\title{
Angiotensin receptor blockers for bipolar disorder
}

\author{
Ana Isabelle de Góis Queiroz, Camila Dantas Medeiros, Bruna Mara Machado Ribeiro, \\ David Freitas de Lucena, Danielle Silveira Macêdo*
}

Neuropharmacology Laboratory, Postgraduate Pharmacology Program, Department of Physiology and Pharmacology, Federal University of Ceará, Fortaleza, Ceará, Brazil Department of Psychiatry, UniChristus Faculty of Medicine, Fortaleza, Ceará, Brazil

\section{A R T I C L E I N F O}

\section{Article history:}

Received 22 August 2012

Accepted 27 November 2012

\begin{abstract}
A B S T R A C T
Studies have suggested that the brain renin angiotensin system (RAS) regulates cerebral flow, autonomic and hormonal systems, stress, innate immune response and behavior, being implicated in several brain disorders such as major depression, Parkinson's and Alzheimer's disease. The angiotensin II receptor subtype 1 (AT1R) is distributed in brain regions responsible for the control of stress response through peripheral and central sympathetic hyperactivation as well as in the hypothalamic paraventricular region, areas known for the release of several neurotransmitters related to inflammatory response facilitation. This relationship leads to the assumption that AT1R might be the receptor most related to the central deleterious actions of angiotensin II. New evidences from clinical studies have shown a possible role for RAS in the pathogenesis of bipolar disorder (BD), a multifactorial disorder with acknowledged presence of neuronal damage via oxidative stress in brain areas such as hippocampus, prefrontal cortex and striatum. Given the studies highlighting AT1R activation as a central pro-inflammatory pathway and, conversely, the involvement of inflammatory response in the pathogenesis of $\mathrm{BD}$; this paper hypothesizes the use of AT1R antagonists for BD management and prevention of its neuroprogression, due to their anti-inflammatory and neuroprotective effects.
\end{abstract}

(ㄷ) 2012 Elsevier Ltd. All rights reserved.

\section{Introduction}

The renin-angiotensin system (RAS) plays an important role in blood pressure regulation and body fluid homeostasis [1]. Renin, produced in the juxtaglomerular cells of the kidney, cleaves an inactive peptide angiotensinogen into angiotensin I. The latter, a precursor of Angiotensin II (AngII) with negligible physiological effects, is converted to AngII by the angiotensin I-converting enzyme (ACE), secreted by pulmonary and renal endothelial cells. Angiotensin II plays a key role in the maintenance of cardiovascular homeostasis regulating blood volume and vascular resistance being therefore involved in the pathophysiology of cardiovascular diseases such as hypertension [2].

The existence of a brain RAS is now widely accepted [3]. This central system includes all the precursors and enzymes required for metabolizing the angiotensins present in the peripheral system. Angiotensin II is widespread in the brain [4]. Previous experiments showed that the injection of AngII into key brain nuclei produced hypertension, an effect blocked by the AngII antagonist saralasin or by the deletion of AngII type 1 receptor (AT1R) [3]. Although

\footnotetext{
* Corresponding author. Address: Department of Physiology and Pharmacology, Federal University of Ceará, Rua Cel. Nunes de Melo 1127, 60431-270 Fortaleza, Ceará, Brazil. Tel.: +55 853366 8337; fax: +55 8533668333 .

E-mail addresses: daniellesilmacedo@gmail.com, daniellesm2000@yahoo.com (D.S. Macêdo).
}

peripheral AngII does not cross the blood-brain barrier (BBB), the communication between peripheral and central RAS is established through circumventricular organs that are sensitive to circulating AngII via ATRs [5]. On the other hand, following peripheral administration, AT1R antagonists, for example telmisartan, can penetrate the BBB in a dose- and time-dependent manner to inhibit centrally mediated effects of AngII [6].

The effects of AngII are usually mediated by two well characterized subtypes of receptors, AT1R and AT2R [7]. These receptors are known to induce $G$ protein- and non-G protein-related signaling pathways. Angiotensin II, via AT1R, carries out its functions via MAP kinases (ERK 1/2, JNK, p38MAPK), receptor tyrosine kinases (PDGF, EGFR, insulin receptor), and nonreceptor tyrosine kinases (Src, JAK/STAT, focal adhesion kinase (FAK)). Of note, alterations in the aforementioned intracellular pathways have been associated with mental disorders [8]. In this sense, MAP kinase-related pathways in the prefronto-striatal circuitries were involved in the manifestation of aggressive behaviors in mice observed after methamphetamine single and multiple injections [9]. Repeated administration of methamphetamine was recently proposed as an animal model of mania [10]. Furthermore, tyrosine hydroxylase activation of the ERK1/2 signal pathway was observed in the ouabain-induced animal model of mania [11]. Concerning the tyrosine kinase signaling pathway, it was previously described that the activity of GSK3 $\alpha / \beta$, an intracellular pathway involved in BD pathophysiology, is positively regulated by phosphorylation on tyrosine 
residues. This phosphorylation is inhibited by lithium, a mood stabilizer drug [12]. In addition, AT1R-mediated NAD(P)H oxidase activation leads to generation of the reactive oxygen species (ROS), widely implicated in vascular inflammation and fibrosis [7], anxiety and mood disorders [13].

The brain contains high densities of AT1Rs mainly localized in specific nuclei within the hypothalamus, brainstem regions as well as hypothalamic-pituitary-adrenal axis (HPA) and amygdala nuclei [14]. Angiotensin II AT1Rs are further subgrouped in the rodent brain into AT1A and AT1B receptors. These are related to osmo-, thermal- and behavioral regulation [15]. Besides the abundant representation of AT1Rs in the adult brain, AT2Rs are expressed in high density mainly in the neonate brain, and stimulation of AT2Rs by AngII provokes apoptosis [15]. Therefore, most of the literature regarding AT2Rs suggests a role in differentiation and development for this receptor. Some of the actions of the AT2Rs are even directly opposed to those of the AT1Rs, especially concerning the growthand differentiation-modulating actions of AngII [16]. Recent studies have highlighted a crucial role of AT2Rs in normal brain function, and the impact of its dysfunction on brain development and ultrastructural morphology with distinct consequences on learning and memory [17]. Thus, there is a consensus that the most damaging effects of AngII in the brain are triggered by AT1Rs activation. Indeed, widespread anti-inflammatory effects of AngII AT1R blockade in the periphery and brain were observed [4]. On the other hand, hyperactivation of these receptors seem to be implicated in mood disorders pathophysiology [18]. Accordingly, an imbalance between the AT1R- and AT2R-triggered signals may lead to diseases such as hypertension [2] and some of the brain dysfunctions seen in mental disorders [13].

It has been previously established that besides the well-known AngII, other angiotensin peptides such as AngIII, AngIV and Ang(1-7) are able to mediate distinct biological effects in the brain. In this way, the heptapeptide Ang-(1-7) has been shown to counterbalance most of the pressoric levels alterations and angiogenic actions of AngII in the periphery [3], and more recently has been shown to be important in the regulation of neuroplasticity and neuroprotection [19].

Overall, the dysregulation of brain RAS is associated with changes in glutamate release, ROS formation and activation of pro-inflammatory pathways [15], events also related to the pathophysiology of mood disorders [20].

Accumulated evidences from clinical [21] and pre-clinical [22] studies have acknowledged the increased levels of lipid peroxidation products and alterations in antioxidant enzymes systems in bipolar disorder (BD). Wang et al. [21] evaluated oxidative stress in the postmortem anterior cingulate brain sections from BD subjects and showed that 4-hydroxynonenal levels, a major product of lipid peroxidation, were significantly increased by $59 \%$ when compared to healthy subjects, suggesting that oxidative damage in the brain may partly contribute to the pathological process in BD [20]. Furthermore, it has been widely demonstrated that the generation of ROS plays a critical role in the pathophysiology of manifold neuropsychiatric disorders [21].

Several lines of evidence have implicated inflammatory abnormalities in BD [20,23]. Accordingly, increased levels of tumor necrosis factor $\alpha$ (TNF- $\alpha$ ), interleukin-1 $\beta$ (IL-1 $\beta$ ) and interleukin 6 (IL-6) have consistently been reported [24]. There seems to be a larger variation in the levels of inflammatory markers in BD patients compared to healthy subjects which further suggests that immune activation could be associated with BD clinical characteristics [20].

Research regarding oxidative and inflammatory mechanisms in BD is still elusive. However, these studies can be useful for the development of new management strategies for this disorder as alternative adjuvant therapy [25]. The measurement of specific biological markers (for example, cytokines) in plasma samples of $\mathrm{BD}$ patients in different mood states, in order to facilitate the diagnosis and treatment outcome of $\mathrm{BD}$, is another important reason for this investigation [26].

Thus, regarding the role of RAS in the regulation of brain inflammatory and oxidative mechanisms, and the involvement of these mechanisms in BD pathophysiology, our hypothesis is that the modulation of brain RAS could be a beneficial add-on treatment for BD management and possible control of its neuroprogression.

\section{The hypothesis}

1. We hypothesize that the blockade of AT1Rs, by controlling pathophysiological alterations related to $\mathrm{BD}$, may be useful as an adjuvant treatment for bipolar mania and depression.

2. Our specific hypothesis is that AT1Rs blockers by the regulation of brain inflammation and oxidative stress could prevent neuronal loss and cognitive impairment in BD contributing, thus, for the maintenance of euthymic state and prevention of neuroprogression, providing a better prognosis for this disorder.

\section{Evaluation of the hypothesis}

One of the first reports correlating the use of the ACE inhibitor, captopril with substantial mood elevation in three depressed patients was published in the early 1980s [27]. Further, cases reporting an improvement in major depression with captopril suggest that altered angiotensin function may play a role in the treatment of depression [28-30]. In more recent years an emphasis on the effects of ACE inhibitors and AT1R blockers against cognitive decline caught the attention for a potential role of these drugs in the prevention or even reversal of vascular dementias and Alzheimer's disease [31]. In this regard, neurochemical studies in animals have shown that AngII is able to inhibit the release of acetylcholine from entorhinal cortex slices. Thus, the ability of ACE inhibitors to facilitate cognitive processes may be related to a decreased availability of AngII [31]. In addition, these drugs also presented antidepressant and anxiolytic effect on experimental and clinical studies [31,32].

Angiotensin-converting enzyme is assumed to influence the activity of the hypothalamic-pituitary-adrenocortical (HPA) system [33], which shows hyperactivity in the majority of patients with major depression [34] and BD [35]. Polymorphisms in the ACE gene were associated with unipolar depression, ACE activity alterations and hypercortisolism probably representing a common pathophysiologic link for unipolar depression and cardiovascular disease [33]. Associations between angiotensinogen and ACE gene polymorphisms and the risk of bipolar affective disorder in humans have been demonstrated as well [36]. In addition, there further appears to be an excess burden of cardiovascular risk factors with BD compared with the general population [37]. One important feature is that patients with co-occurring disorders, for example, cardiovascular disease and BD, experience worse prognosis with less favorable response to treatment, unemployment and thus higher cost than those without comorbidity. Moreover cardiovascular mortality is known to be a leading cause of excess mortality in BD, well above the risk associated with un-natural causes of death such as suicide or accidents [23].

Recently it was demonstrated that astrocytes, microglia, and neurons express AT1R on high levels and respond to its activation by initiating the upregulation and activation of TGF- $\beta$, a highly pleiotropic and multifunctional molecule that plays pivotal role in the immune response [38]. Accordingly, AngII AT1R blockers exhibit anti-inflammatory effects not only in periphery of the body, but also in the brain [4]. Following this line of reasoning, recent 
research has highlighted the effects of AT1R blockers in improving inflammatory stress, being therefore beneficial for the treatment of brain disorders in which inflammation is a common feature [3941]. Over the last years, it has been shown that inflammatory mechanisms and neural immune interactions are involved in the pathophysiology of psychiatric disorders such as major depression [42] and BD [20]. Accordingly, evidence reveals that chronic, mild inflammation in the periphery and in the brain occurs during BD [23]. This data provides a plausible explanation for the increased levels of pro-inflammatory cytokines in BD. Indeed, increased IL-1, IL- 6 and TNF- $\alpha$ have been reported at differing stages of the illness [20]. This pro-inflammatory state is known to activate the tryptophan and serotonin degrading enzyme, indoleamine 2-3 dioxygenase (IDO), which has been found elevated in the plasma of bipolar patients [43]. IDO activation leads to increased consumption of tryptophan, the substrate for serotonin synthesis, thus reducing serotonergic neurotransmission and inducing the production of detrimental tryptophan catabolites (also called TRYCAT),

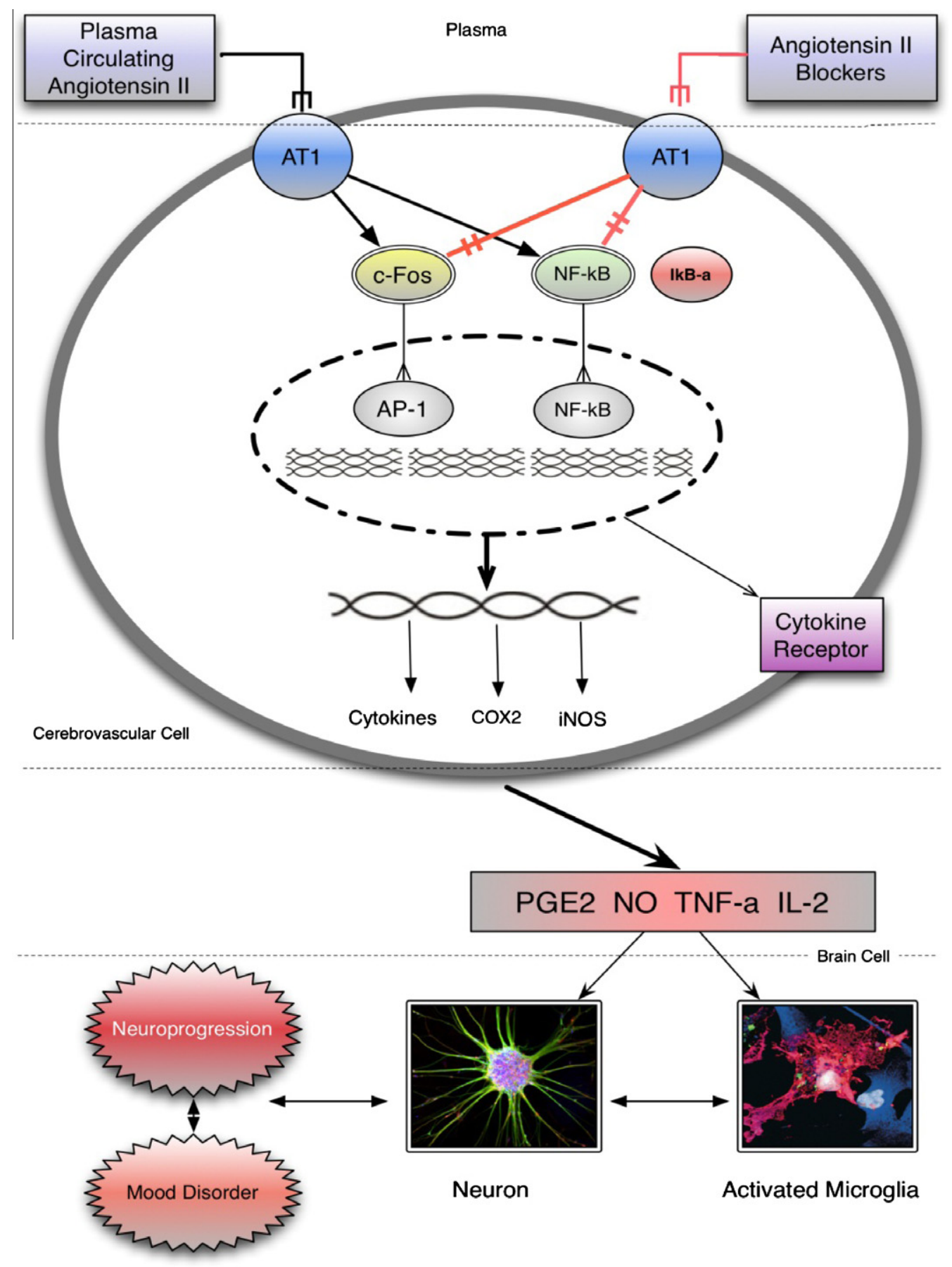

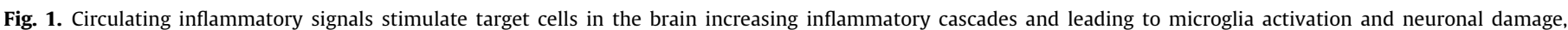

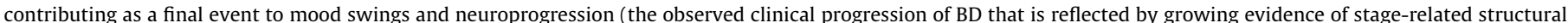

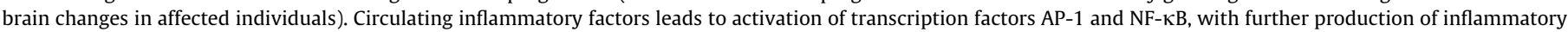

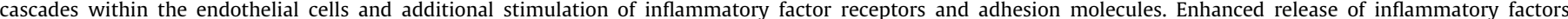

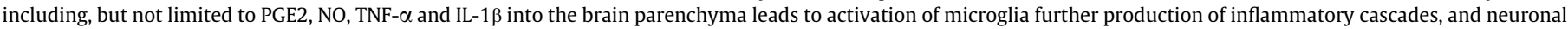
injury. Adapted from Saavedra et al. [41] and Berk et al. [20]. 
such as kynurenic acid, which present neurotoxic effects [43]. IDO activity is also altered by cortisol [43]. Currently, dysregulation of the hypothalamic-pituitary-adrenal (HPA) axis is thought to be associated with mood symptoms and worse cognitive functioning, although the researches in this field are still inconclusive [44]. As far as we know there are no studies evaluating the effects of AT1R blockers on TRYCAT generation. Meanwhile, regarding the HPA axis, the pretreatment with the AT1R blocker, candesartan, prevented the effects of isolation stress on HPA axis activation [45].

Pro-inflammatory cytokines are also able to induce oxidative and nitrosative stress pathways, which can damage lipids, DNA and proteins, cause mitochondrial dysfunction and consequently apoptosis as well as cell membrane damage and protein aggregation. Stage dependent changes in oxidative stress have been reported in late stage more than in early stage bipolar patients, which may be part of a progressive failure of compensatory mechanisms over time, leading to cognitive decline and resistant $\mathrm{BD}$, what may in part underlie the staging process [20]. In line with the aforementioned evidences for the involvement of oxidative stress and mitochondrial dysfunction in BD, valsartan, an AT1R blocker, exhibited neuroprotective effects on ischemic injury through the suppression of oxidative stress and mitochondrial injury [46]. Conversely, antioxidant drugs [25] and mitochondrial modulators [47] present mood stabilizer properties.

Angiotensin II seems to regulate dopamine neurotransmission as well [48]. In BD pathophysiology the dopamine hypothesis is related to the presence of a dopamine dysregulation syndrome underlying this disorder. According to this hypothesis an increased dopaminergic drive is related to symptoms of mania and the converse to depression [49]. Dopamine brain levels are influenced by angiotensin, because AngII receptors are found on the soma and terminals of mesolimbic dopaminergic neurons and within this dopaminergic pathway AngII binding to AT1Rs facilitates dopamine release. Indeed, in vitro pretreatment with candesartan, an AT1R blocker was able to attenuate dopamine release induced by amphetamine in caudate-putamen and nucleus accumbens slices showing, thus, that AT1R activation is related to the neuroadaptative changes induced by amphetamine [48]. Of note, the repeated amphetamine administration is broadly used as an animal model of mania [22,50].

It was previously demonstrated that pro-inflammatory cytokines decrease neurotrophin levels, particularly brain derived neurotrophic factor (BDNF), what leads to impairment in neuronal repair and neurogenesis and to activation of glutamatergic pathways (which also contributes to neuronal apoptosis). This mechanism is associated with changes in mood states in BD [23]. Indeed, the AT1R blocker telmisartan protects against cognitive decline via up-regulation of BDNF/tropomyosin-related kinase B in the hippocampus of hypertensive rats [51]. Similar effect (i.e. BDNF upregulation) was demonstrated for candesartan [52].

Excessive allostatic load, a phenomenon triggered by long time intervals of stress response together with glucocorticoids and catecholamines release alterations, is known to be related to the development and neuroprogression of multiple brain diseases, including, but not limited to, mood disorders [53,54]. Thus, in our hypothesis inhibition of the peripheral and brain RAS by the administration of AngII AT1Rs blockers could prevent allostatic load by preventing pro-inflammatory alterations associated with mood swings as well as other brain alterations related to BD pathophysiology because, as previously mentioned, chronic, mild inflammation in the periphery and in the brain occurs during BD [23] (Fig. 1). Other pathophysiological mechanisms related to BD that can likely be regulated by AT1R blockers administration include BDNF decrease, hypercortisolism and oxidative stress. Thus, our hypothesis for the use of AT1Rs blockers in BD management is supported by six arguments: (i) decrease of inflammatory mark- ers in peripheral organs and their release to the circulation; (ii) reduced progression of peripherally induced inflammatory cascades in the cerebral vasculature and brain parenchyma; (iii) direct anti-inflammatory effects in cerebrovascular endothelial cells, microglia, and neurons; (iv) antioxidant effects; (v) upregulation of BDNF levels; (vi) adjustment of dopamine levels. Accordingly, the clinical use of these drugs may provide a mood stabilizing effect accompanied by reduction or prevention in its neuroprogression and cognitive decline.

One possible limitation that needs to be addressed in the upcoming clinical trials is the drug interaction between lithium and AT1R blockers, what results in an increased risk for lithium intoxication [55]. Thus, much care must be taken when prescribing these drugs.

Taken as a whole, we propose that the accomplishment of preclinical and clinical studies regarding the use of AT1Rs blockers as adjuvant (add-on) therapies for BD is of immediate translational relevance.

\section{Conclusions and future perspectives}

The AT1Rs blockers play an important role in the regulation of blood pressure and current research has demonstrated its benefits in neuropsychiatric disorders, such as Alzheimer's [31], Parkinson's [41] and major depression [30]. Brain RAS is related to pro-inflammatory mechanisms which mainly affects regions responsible for the emotion, as the limbic system, hippocampus, hypothalamus and prefrontal cortex [14]. In line with this evidence, therapy with AT1Rs blockers can be a new pharmacological strategy to prevent and/or treat BD patients, based on the relation of neuroinflammation and oxidative stress with the pathophysiology of this disorder [20]. Thus, this paper proposes the development of future pre-clinical researches to evaluate the antimanic effect of these drugs and the possible mechanism of action, as well as clinical researches to determine its therapeutic profile in $\mathrm{BD}$ patients, opening therefore new avenues for the treatment of this mental disorder already known to be closely related to cardiovascular disease comorbidity [23]. Our hypothesis has one limitation. Inhibitors of AT1Rs may raise lithium levels increasing, thus, the risks of lithium toxicity [55]. Thereby, great importance should be given to this event because lithium is a first-line drug in the treatment of $\mathrm{BD}$.

\section{Conflict of interest statement}

None declared.

\section{References}

[1] Ito M, Oliverio MI, Mannon PJ, et al. Regulation of blood-pressure by the type 1A angiotensin-II receptor gene. Proc Natl Acad Sci U S A 1995;92:3521-5.

[2] de Gasparo M, Catt KJ, Inagami T, Wright JW, Unger T. International union of pharmacology. XXIII. The angiotensin II receptors. Pharmacol Rev 2000;52:415-72.

[3] Phillips MI, de Oliveira EM. Brain renin angiotensin in disease. J Mol Med-Jmm 2008;86:715-22.

[4] Benicky J, Sanchez-Lemus E, Pavel J, Saavedra JM. Anti-inflammatory effects of angiotensin receptor blockers in the brain and the periphery. Cell Mol Neurobiol 2009;29:781-92.

[5] Phillips MI. Functions of angiotensin in the central-nervous-system. Annu Rev Physiol 1987;49:413-35.

[6] Gohlke P, Weiss S, Jansen A, et al. AT(1) receptor antagonist telmisartan administered peripherally inhibits central responses to angiotensin II in conscious rats. J Pharmacol Exp Therapeut 2001;298:62-70.

[7] Mehta PK, Griendling KK. Angiotensin II cell signaling: physiological and pathological effects in the cardiovascular system. Am J Physiol Cell Physiol 2007;29(2):26

[8] Dwivedi Y, Rizavi HS, Roberts RC, Conley RC, Tamminga CA, Pandey GN. Reduced activation and expression of ERK1/2 MAP kinase in the post-mortem brain of depressed suicide subjects. J Neurochem 2001;77:916-28. 
[9] Sokolov BP, Cadet JL. Methamphetamine causes alterations in the MAP kinaserelated pathways in the brains of mice that display increased aggressiveness. Neuropsychopharmacology 2006;31:956-66.

[10] Feier G, Valvassori SS, Varela RB, et al. Lithium and valproate modulate energy metabolism in an animal model of mania induced by methamphetamine. Pharmacol Biochem Behav.

[11] Yu HS, Kim SH, Park HG, Kim YS, Ahn YM. Intracerebroventricular administration of ouabain, a $\mathrm{Na} / \mathrm{K}$-ATPase inhibitor, activates tyrosine hydroxylase through extracellular signal-regulated kinase in rat striatum. Neurochem Int 2011;59:779-86.

[12] Gould TD, Zarate CA, Manji HK. Glycogen synthase kinase-3: a target for novel bipolar disorder treatments. J Clin Psychiatry 2004;65:10-21.

[13] Liu F, Havens J, Yu Q, et al. The link between angiotensin II-mediated anxiety and mood disorders with NADPH oxidase-induced oxidative stress. Int J Physiol Pathophysiol Pharmacol 2012;4:28-35.

[14] Karamyan VT, Speth RC. Distribution of the non-AT1, non-AT2 angiotensinbinding site in the rat brain: preliminary characterization. Neuroendocrinology 2008;88:256-65.

[15] Albrecht D. Physiological and pathophysiological functions of different angiotensins in the brain. Br J Pharmacolgy 2010;159:1392-401.

[16] Carey RM, Padia SH. Angiotensin AT2 receptors: control of renal sodium excretion and blood pressure. Trends Endocrinol Metabol 2008;19:84-7.

[17] Horiuchi M, Mogi M. Role of angiotensin II receptor subtype activation in cognitive function and ischaemic brain damage. $\mathrm{Br} J$ Pharmacol 2011;163:1122-30.

[18] Saavedra JM, Benicky J, Zhou J. Angiotensin II: multitasking in the brain. J Hypertens 2006;24:S131-7.

[19] Davisson RL, Oliverio MI, Coffman TM, Sigmund CD. Divergent functions of angiotensin II receptor isoforms in the brain. J Clin Invest 2000;106:103-6.

[20] Berk M, Kapczinski F, Andreazza AC, et al. Pathways underlying neuroprogression in bipolar disorder: focus on inflammation; oxidative stress and neurotrophic factors. Neurosci Biobehav Rev 2011;35:804-17.

[21] Wang J-F, Shao L, Sun X, Young LT. Increased oxidative stress in the anterior cingulate cortex of subjects with bipolar disorder and schizophrenia. Bipolar Disord 2009;11:523-9.

[22] Frey BN, Valvassori SS, Reus GZ, et al. Changes in antioxidant defense enzymes after D-amphetamine exposure: implications as an animal model of mania. Neurochem Res 2006;31:699-703.

[23] Leboyer M, Soreca I, Scott J, et al. Can bipolar disorder be viewed as a multisystem inflammatory disease? J Affect Disord 2012;141:1-10.

[24] Brietzke E, Stertz L, Fernandes BS, et al. Comparison of cytokine levels in depressed, manic and euthymic patients with bipolar disorder. J Affect Disord 2009;116:214-7.

[25] Macedo DS, Medeiros CD, Cordeiro RC, et al. Effects of alpha-lipoic acid in an animal model of mania induced by d-amphetamine. Bipolar Disord 2012;17:1399-5618.

[26] Berk M, Kapczinski F, Andreazza AC, et al. Pathways underlying neuroprogression in bipolar disorder: focus on inflammation, oxidative stress and neurotrophic factors. Neurosci Biobehav Rev 2011;35:804-17.

[27] Zubenko GS, Nixon RA. Mood-elevating effect of captopril in depressed patients. Am J Psychiatry 1984;141:110-1.

[28] Germain L, Chouinard G. Treatment of recurrent unipolar major depression with captopril. Biol Psychiatry 1988;23:637-41.

[29] Cohen BM, Zubenko GS. Captopril in the treatment of recurrent major depression. J Clin Psychopharmacol 1988 Apr;8(2):143-4.

[30] Gard PR, Mandy A, Sutcliffe MA. Evidence of a possible role of altered angiotensin function in the treatment, but not etiology, of depression. Biol Psychiatry 1999;45:1030-4.

[31] Gard PR. Angiotensin as a target for the treatment of Alzheimer's disease, anxiety and depression. Expert Opin Ther Targets 2004;8:7-14.

[32] Hertzman M, Adler LW, Arling B, Kern M. Lisinopril may augment antidepressant response. J Clin Psychopharmacol 2005 Dec;25(6):618-20.

[33] Baghai TC, Binder EB, Schule C, et al. Polymorphisms in the angiotensinconverting enzyme gene are associated with unipolar depression, ACE activity and hypercortisolism. Mol Psychiatry 2006;11:1003-15.
[34] Pariante CM, Lightman SL. The HPA axis in major depression: classical theories and new developments. Trends Neurosci 2008;31:464-8.

[35] Watson S, Gallagher P, Ritchie JC, Ferrier IN, Young AH. Hypothalamicpituitary-adrenal axis function in patients with bipolar disorder. $\mathrm{Br} \mathrm{J}$ Psychiatry 2004:184:496-502.

[36] Meira-Lima IV, Pereira AC, Mota GFA, Krieger JE, Vallada H. Angiotensinogen and angiotensin converting enzyme gene polymorphisms and the risk of bipolar affective disorder in humans. Neurosci Lett 2000;293:103-6.

[37] Birkenaes AB, Opjordsmoen S, Brunborg C, et al. The level of cardiovascular risk factors in bipolar disorder equals that of schizophrenia: a comparative study. J Clin Psychiatry 2007;68:917-23.

[38] Lanz TV, Ding Z, Ho PP, et al. Angiotensin II sustains brain inflammation in mice via TGF-beta. J Clin Invest 2010;120:2782-94.

[39] Saavedra JM. Angiotensin II AT(1) receptor blockers ameliorate inflammatory stress: a beneficial effect for the treatment of brain disorders. Cell Mol Neurobiol 2012;32:667-81.

[40] Saavedra JM. Angiotensin II AT(1) receptor blockers as treatments for inflammatory brain disorders. Clin Sci 2012;123:567-90.

[41] Saavedra JM, Sanchez-Lemus E, Benicky J. Blockade of brain angiotensin II AT1 receptors ameliorates stress, anxiety, brain inflammation and ischemia: Therapeutic implications. Psychoneuroendocrinology 2011;36:1-18.

[42] Maes M, Berk M, Goehler L, et al. Depression and sickness behavior are Janusfaced responses to shared inflammatory pathways. BMC Med 2012;1:66.

[43] Anderson G, Maes M, Berk M. Inflammation-related disorders in the tryptophan catabolite pathway in depression and somatization In: Donev R, Editor. Inflammation in neuropsychiatric disorders vol. 88. 2012; pp. 27-48.

[44] van der Werf-Eldering MJ, Riemersma-van der Lek RF, Burger H, Holthausen EAE, Aleman A, Nolen WA. Can variation in hypothalamic-pituitary-adrenal (HPA)-axis activity explain the relationship between depression and cognition in bipolar patients? Plos One 2012;7.

[45] Armando I, Volpi S, Aguilera G, Saavedra JM. Angiotensin II AT1 receptor blockade prevents the hypothalamic corticotropin-releasing factor response to isolation stress. Brain Res 2007;1142:92-9.

[46] Wakai T, Yoshioka H, Yagi T, Kato T, Kinouchi H. Effects of valsartan on neuroprotection and neurogenesis after ischemia. Neuroreport 2011;22:385-90.

[47] Nierenberg AA, Kansky C, Brennan BP, Shelton RC, Perlis R, Iosifescu DV. Mitochondrial modulators for bipolar disorder: a pathophysiologically informed paradigm for new drug development. Aust N Z J Psychiatry 2012;1(8):18.

[48] Paz MC, Assis MA, Cabrera RJ, Cancela LM, Bregonzio C. The AT(1) angiotensin II receptor blockade attenuates the development of amphetamineinduced behavioral sensitization in a two-injection protocol. Synapse 2011;65:505-12

[49] Berk M, Dodd S, Kauer-Sant'anna M, et al. Dopamine dysregulation syndrome: implications for a dopamine hypothesis of bipolar disorder. Acta Psychiatr Scand Suppl 2007;434:41-9.

[50] Frey BN, Valvassori SS, Reus GZ, et al. Effects of lithium and valproate on amphetamine-induced oxidative stress generation in an animal model of mania. J Psychiatry Neurosci 2006;31:326-32.

[51] Kishi T, Hirooka Y, Sunagawa K. Telmisartan protects against cognitive decline via up-regulation of brain-derived neurotrophic factor/tropomyosin-related kinase B in hippocampus of hypertensive rats. J Cardiol 2012;1:1.

[52] Krikov M, Thone-Reineke C, Muller S, Villringer A, Unger T. Candesartan but not ramipril pretreatment improves outcome after stroke and stimulates neurotrophin BNDF/TrkB system in rats. J Hypertens 2008;26:544-52.

[53] Karatsoreos IN, McEwen BS. Psychobiological allostasis: resistance, resilience and vulnerability. Trends Cogn Sci 2011;15:576-84.

[54] Kapczinski F, Vieta E, Andreazza AC, et al. Allostatic load in bipolar disorder: implications for pathophysiology and treatment. Neurosci Biobehav Rev 2008;32:675-92.

[55] Zwanzger P, Marcuse A, Boerner RJ, Walther A, Rupprecht R. Lithium intoxication after administration of AT1 blockers. J Clin Psychiatry 2001 Mar;62(3):208-9. 